\title{
Alpha-Solanine Anti-Tumor Effects in Non-Small Cell Lung Cancer Through Regulating the Glycolysis Pathway
}

\section{Tao Zou}

Southwest Medical University

\section{Yuankai Si}

Southwest Medical University

Yifan Hu

Southwest Medical University

\section{Liqiong Yang}

Southwest Medical University

Junsong Wei

Southwest Medical University

Junxin Li

Southwest Medical University

Yueshui Zhao

Southwest Medical University

Jing Shen

Southwest Medical University

Mingxing Li

Southwest Medical University

$\mathrm{Xu} \mathrm{Wu}$

Southwest Medical University

\section{Fukuan Du}

Southwest Medical University

\section{Yu Chen}

Southwest Medical University

\section{Meijuan Chen}

Southwest Medical University

\section{Yun Ye}

Affiliated Hospital of Southwest Medical University

\section{Zhangang Xiao}

Southwest Medical University

Zhigui Wu ( $\square$ zhiguiwu18@126.com ) 


\section{Research Article}

Keywords: NSCLC, a-solanine, Network Pharmacology, Metabolomics, Glycolyticpathway

Posted Date: August 13th, 2021

DOl: https://doi.org/10.21203/rs.3.rs-789534/v1

License: (c) (i) This work is licensed under a Creative Commons Attribution 4.0 International License. Read Full License 


\section{Abstract}

Background: Lung cancer is a malignant tumor with a high incidence in China, especially non-small cell lung cancer (NSCLC), which is the main threat to human life, with terrible morbidity and mortality. The research on the treatment and mechanism of lung cancer and NSCLC has been the forefront and hotspot of research. Alpha-solanine (a-solanine) has anti-tumor effect, but its target and related mechanism remain to be elucidated.

Methods: Network pharmacology was used to predict the possible targets and mechanisms of a-solanine on NSCLC. The targets were derived from TGCC database, and a-solanine targets were derived from PharmMapper. Gene-related potential pathways were identified by gene ontology and pathway enrichment analysis. Compound-target and target-pathway networks were constructed and validated by molecular biology and targeted energy metabolomics. Experiments were used to verify the results of network pharmacology in vitro, such as cell migration and invasion, cell apoptosis, immunofluorescenceand western blot.

Results: Network pharmacology showed that there were 130 potential targets of a-solanine and NSCLC, and the main core was the pathway of glycolysis. In addition, experiment results showed that a-solanine inhibited cell proliferation, migration, invasion and promoted cell apoptosis. At the same time, a-solanine mainly regulated cell proliferation and survival throughglycolytic signaling pathway, which included GPI, ALDOA, TPI1, PKLR, LDHA and ALDH 3 . After a-solanine treatment, the expression of the above-mentioned proteins was reduced.

Conclusion: Combination of network pharmacological prediction and experimental verification, a-solanine affected the expression of related proteins and cell functions by regulating the glycolytic pathway, thereby achieving anti- NSCLC effects.

\section{Introduction}

Lung cancer remains the leading cause of cancer-related deaths worldwide ${ }^{[1]}$. There are two main forms of lung cancer: small cell lung cancer and non-small cell lung cancer (NSCLC). NSCLC is the most common histological subtype, accounting for about $85 \%$ of all lung cancer cases. Compared with small cell lung cancer, the growth and division of NSCLC cells are slower, and the spread and metastasis are late ${ }^{[2]}$. However, NSCLC is not diagnosed until advanced disease appears. In $50-75 \%$ of patients, cough is the most common symptom, followed by hemoptysis, chest pain, and dyspnea ${ }^{[3]}$. The current treatments for NSCLC mainly include: (1) surgical treatment (2) chemotherapy (3) radiotherapy ${ }^{[4]}$ (4) immunotherapy ${ }^{[2,5]}(5)$ targeted therapy ${ }^{[6]}$. However, how to control the development of lung cancer and explore effective treatment methods are the main problems that need to be solved. Although targeted therapy is a popular direction for lung cancer, which has achieved curative effects in special populations, traditional chemotherapy or radiotherapy is still the main choice. Therefore, the development of new 
strategies to inhibit the growth of NSCLC cells and enhance the sensitivity of chemotherapy and radiotherapy is the focus of current medical research.

Solanine, also known as solanine toxin, and potato toxin, is a weakly basic glycoside composed of glucose residues and solanidine. It is widely found in Solanaceae plants such as potatoes, tomatoes and eggplants ${ }^{[7]}$. The a-solanine is the main biologically active component in solanine. What is more, asolanine has shown anti-tumor cell metastasis activity in a number of tumor treatment experiments ${ }^{[8]}$. In recent years, more and more research showed that a-solanine had anti-proliferation effect on various human tumor cells in vitro ${ }^{[9]}$. The main anti-tumor mechanisms of a-solanine are: (1) inhibit cancer cell proliferation and metastasis ${ }^{[10,11]}(2)$ cytotoxic effect $^{[12,13]}$ (3) induce tumor cell apoptosis ${ }^{[14]}$ (4) inhibit the invasion of cancer cells into tissues ${ }^{[15]}(5)$ enhance the sensitivity of tumor cells to radiotherapy and chemotherapy ${ }^{[16,17]}$ (6) Enhance immunity ${ }^{[18]}$. Some scholars believed that a-solanine can play an anticancer effect in lung cells by inducing the expression of microRNA-138 (MIR-138). In addition, studies have addressed that a-solanine enhanced the chemotherapy sensitivity and radiosensitivity of A549 and H1299 cells by affecting the expression of MIR-138 and focal adhesion kinase (FAK). This indicated that the a-solanine/MIR-138/FAK cascade may be a potential target for the treatment of NSCLC ${ }^{[19]}$. Lin et al. explored that a-solanine inhibited the proliferation and migration of HepG2 cells induced by acetylcholine signal. They also demonstrated that a-solanine has the potential to inhibit liver cancer metastasis by regulating epithelial-mesenchymal transition (EMT), exosomes and miR-21 ${ }^{[20]}$. Acetylcholine can bind to niacin and physostigmine receptors on lung cancer cells, thereby accelerating their proliferation, migration and invasion ${ }^{[21]}$. Whereas, there is no research reported on the therapeutic effect of a-solanine on lung cancer and its related molecular mechanisms, the mechanism of a-solanine on NSCLC is still unclear. Therefore, the target of a-solanine, as well as the metabolite changes and metabolic pathways of a-solanine after acting on the body need to be further studied.

Based on the rapid development of omics and big data, network pharmacology integrates multiple emerging interdisciplinary disciplines such as systems biology and bioinformatics. It is an overall analysis of biological system network ${ }^{[22]}$. Network pharmacology techniques can combine drug action networks with biological networks ${ }^{[23]}$. Network pharmacology can quickly and efficiently predict and screen the effective components of traditional Chinese medicine (TCM), and analyze the possible interactions between components to explore the possibility of drug regulation ${ }^{[24]}$. Therefore, this study used network pharmacology to predict the targets of solanine acting on NSCLC and establish a multilevel "drug targets - disease" network. Then, bioinformatics methods were used to analyze the pathways and mechanisms corresponding to the target. According to the results of the analysis of network pharmacology validated using targeted metabolomics. Finally, the molecular biological experiments were used to verify the mechanism of a-solanine on NSCLC, and to provide the basis for the rational application and secondary development of a-solanine. The detailed technical strategy for the current study is shown in Fig. 1. 


\section{Materials And Methods}

\section{Network Pharmacology Analysis}

\section{Prediction of Drug Targets for a-solanine}

Molecular file of a-solanine were downloaded from Pubchem (https://pubchem.ncbi.nlm.nih.gov/). Then the targets of the constituents were collected from PharmMapper (http://www.lilabecust.cn/pharmmapper/submitfile.html). The obtained targets were imported into UniProt (https://www.uniprot.org/) to search their official gene.

\section{Collection And Analysis Of Gene Targets For Nsclc}

The genes of NSCLC patients were obtained from the TCGA database (https://portal.gdc.cancer.gov). Differentially expressed gene analysis was performed in R software. Genes with a $P$ value $<0.05$ and $\mid \log 2$ (fold change) | > 2 were considered to be of significantly differential expression between NSCLC tissue samples and normal samples. The R package "DEseq2" was used for differential expression analysis. The heatmap and volcano plot were drawn with "pheatmap" R package and "ggplot2" R package.

\section{Screening of candidate targets and construction of network}

The intersection targets of a-solanine and NSCLC were obtained by Venn tool(http://bioinformatics.psb.ugent.be/webtools/Venn/), namely the candidate genes of a-solanine for NSCLC targets. Then Cytoscape software (ver. 3.8.1) was used to construct drug and disease target network diagram to show the relationship between a-solanine and NSCLC.

\section{Construction Of Ppi Network And Selection Of Core Genes}

The obtained candidate targets of a-solanine and NSCLC were uploaded to online STRING11.0 (https://www.string-db.org/) to obtain forecasted protein-protein interactions (PPI), with the species set as "Homo sapiens". Then download the PPI network file and import it into Cytoscape for topology attribute analysis. CytoNCA was used to calculate degree centrality (DC), median centrality (BC), proximity centrality (CC), eigenvector centrality (EC), network centrality (NC) and local mean connectivity (LAC). Based on the results above the median of the six parameters, the core target of the network was obtained.

\section{Gene Ontology and Pathway Enrichment Analysis for NSCLC-Related Targets of a-solanine}

The candidate targets of a-solanine and NSCLC were bioinformatics analyzed by R software. Gene Ontology (GO) function enrichment and KEGG pathway analysis were performed using BiocManager, Dose, Cluster Profiler and Enrichment Plot in R software. The top 20 items with the highest degree were presented in the form of bubble diagram. 


\section{Experimental Validation}

\section{Cell Culture and Drug Preparation}

Human Lung cancer A549 and PC-9 cells were obtained from the Type Culture Collection of the Chinese Academy of Sciences. The cells were cultured in RPMI-1640 medium (Gibco, Thermo Fisher Scientific, Inc.) with $10 \%$ fetal bovine serum (FBS, Gibco, Thermo Fisher Scientific, Inc.) and $1 \%$ streptomycin and penicillin (Gibco), and maintained at $37^{\circ} \mathrm{C}$ in a humidified atmosphere containing $5 \% \mathrm{CO}_{2}$. The a-solanine (J \& K Scientific) were dissolved in dimethyl sulfoxide (DMSO, Sigma-aldrich). The control group was treated with DMSO only under identical conditions.

\section{Cell Viability Assay}

In this study, $3 \times 10^{3}$ lung cancer cells were inoculated into 96 -well plates, and $0,10,12,14,16,18,20,22$, $24 \mathrm{\mu g} / \mathrm{mL}$ concentrations of a-solanine with the same volume of DMSO were added after $24 \mathrm{~h}$; At different time points during treatment, $10 \mu$ l CCK-8 (Dojindo) solution was added to the wells and incubated at $37^{\circ} \mathrm{C}$ for $2 \mathrm{~h}$, and the optical density (OD) was measured by a plate reader (SpectraMax M5; Molecular Devices LLC) at the read mode of absorbance $(450 \mathrm{~nm})$.

\section{Cell Migration And Invasion Assays}

For cell migration assays, transwell filters (Costar) were uncoated with Matrigel on the upper surface of a polycarbonic membrane ( $6.5 \mathrm{~mm}$ diameter, $8 \mu \mathrm{m}$ pore size). After being cultured for $24 \mathrm{~h}$ in medium containing various concentrations of a-solanine $(0,12,18$ and $24 \mu \mathrm{g} / \mathrm{mL}), A 549$ and PC-9 cells $\left(2 \times 10^{5}\right)$ were resuspended in $200 \mu \mathrm{L}$ of serum-free medium in the upper chambers, while $600-\mu \mathrm{L}$ medium containing $10 \%$ FBS (as a chemoattractant) was added to the bottom chamber. After incubating for $24 \mathrm{~h}$ at $37^{\circ} \mathrm{C}$ in a humidified incubator with $5 \% \mathrm{CO}_{2}$, cells in the upper chamber were carefully removed with a cotton swab. Cells that had migrated to the basal side of the membrane were fixed with $4 \%$ polyformaldehyde, stained with crystal violet, mounted and dried at room temperature for 30 minutes. Then randomly selected three visual fields and counted and recorded the number of cells invading the Matrigel $(3.9 \mu \mathrm{g} / \mu \mathrm{L}, 60-80 \mu \mathrm{L})$ with an inverted microscope at $\times 100$ magnification. Each test was performed in triplicate. With a similar principle and approach, we used Transwell filters with coated Matrigel on the upper surface of a polycarbonic membrane for a cell invasion assay.

\section{Apoptosis Analysis By Flow Cytometry (Fcm)}

Apoptosis was detected according to the manufacturer's instructions. Briefly, $3 \times 10^{5}$ A549 and PC-9 cells were inoculated into 6-well plates, after being cultured for $24 \mathrm{~h}$, then treated with $0,12,18,24 \mu \mathrm{g} / \mathrm{mL}$ concentrations of a-solanine with the same volume of DMSO. After $24 \mathrm{~h}$ of treatment, the cells were 
collected and washed with phosphate buffered saline (PBS), resuspended in binding buffer, labeled by Annexin V-FITC, incubated with propidium (PI), and apoptosis was detected by flow cytometry.

\section{The Detection Of Targeted Energy Metabolite}

After treated with a-solanine for 24 hours, A549 cells were collected and extracted with $80 \%$ methanol [25] The supernatant was removed and analyzed by LC-MS/MS system (HPLC, Shim-pack UFLC SHIMADZU CBM30A system, MS/MS, Tandem mass spectrometry, Qtrap ${ }^{\circledR 6500+)}$. Samples were separated using SeQuant ZIC-pHILIC $(5 \mu \mathrm{m}, 2.1 \times 100 \mathrm{~mm})$ at $40^{\circ} \mathrm{C}$. Mobile phases were made up of mobile phase $\mathrm{A}(10$ $\mathrm{mmol} / \mathrm{L}$ ammonium acetate $+0.3 \%$ ammonia solution,) and mobile phase B ( $90 \%$ acetonitrile water). The elution gradient was as follows: 5:95 V/V at $0 \mathrm{~min}, 50: 50 \mathrm{~V} / \mathrm{V}$ at $9.5 \mathrm{~min}, 5: 95 \mathrm{~V} / \mathrm{V}$ at $11.1 \mathrm{~min}, 5: 95 \mathrm{~V} / \mathrm{V}$ at

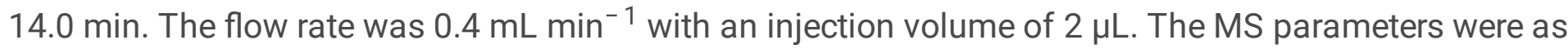
follows: electrospray ionization temperature: $450^{\circ} \mathrm{C}$; mass voltage: $5500 \mathrm{~V}$ (positive ion mode) or $-4500 \mathrm{~V}$ (negative ion mode); ion source gas: GS I 40 psi, GS II 55 psi; curtain gas: 35 psi; collision-activated dissociation: medium. The mass detection was optimized based on declustering potential and collision energy.

\section{Immunofluorescent Staining}

A549 and PC-9 cells $\left(2 \times 10^{5}\right)$ were inoculated into 6-well plate (Corning), after being cultured for $24 \mathrm{~h}$ in order to make the cells adhere and stabilize. Then the test group treated with a-solanine $(18 \mu \mathrm{g} / \mathrm{mL})$, an equal volume of DMSO was used as control. After $24 \mathrm{~h}$ of treatment, the cells were collected and washed with PBS, then cells were fixed with $4 \%$ polyformaldehyde, and permeabilized with $0.1 \%$ Triton X-100 made in PBS for 15-20 minutes. Prepare a blocking solution of 5\% normal goat serum in PBS for 30 minutes, then incubated with relevant antibodies (Dilute with $5 \%$ goat serum) overnight at $4{ }^{\circ} \mathrm{C}$. Apply an appropriate fluorophore-conjugated secondary antibody diluted in antibody dilution buffer to the coverslips and incubate for 1 hour in a moist, dark environment. Mount cover slips on microscope slides with Hydromount (National Diagnostics) containing DAPI (if desired) for nuclear staining.

\section{Protein Extraction And Western Blot Assay}

In brief, A549 and PC-9 cells were treated with $0,12,18$ and $24 \mu \mathrm{g} / \mathrm{mL}$ a-solanine (final concentration) with the same volume of DMSO for $24 \mathrm{~h}$, collected, lysed with radioimmune precipitation assay (RIPA) buffer (Beyotime Institute of Biotechnology), quantified by BCA Protein Assay Kit (Beyotime Institute of Biotechnology), and separated by $10 \%$ SDS-PAGE. The proteins were transferred onto $0.22 \mu \mathrm{m}$ PVDF membrane (Bio-Rad). The membranes were then blocked with $5 \%$ non-fat milk for 1 hour at room temperature, then incubated with relevant antibodies overnight at $4^{\circ} \mathrm{C}$ against GPI $(1: 1,000$, Proteintech, 15171-1-AP), ALDOA (1:1,000, Proteintech, 11217-1-AP), TPI1 (1:1,000, Proteintech, 10713-1-AP), PKLR (1:1,000, Proteintech, 22456-1-AP), LDHA (1:1,000, Proteintech, 19987-1-AP), ALDH 3 (1:1,000, Proteintech, 
15578-1-AP) and GAPDH (1:3,000, GENE TEX, GTX100118), washed with PBST (0.1\% Tween-20, Diluted with PBS). The blots were then incubated with horseradish peroxidase (HRP)-conjugated secondary antibodies (goat anti-rabbit IgG, 1:3000, Beyotime, A0516) and visualized by an enhanced chemiluminescence substrate (Thermo Fisher Scientific, Inc.). Band density were evaluated by ImageJ (National Institutes of Health).

\section{Statistical Analysis}

Statistical analysis was processed with GraphPad Prism 8 software. Data were expressed as the mean \pm SD and analyzed using Student's t-test (for comparison between two groups) or one-way ANOVA with a post hoc Tukey test (for comparison among three or more groups). Differences between groups were considered to be statistically significant if values of $P<0.05$.

\section{Results}

\section{Network Pharmacology Analysis}

\section{Results of genes in NSCLC and target genes of a-solanine}

From TCGA data analysis,7845 differentially expressed genes were found in NSCLC, of which 5343 genes were up-regulated and 2502 genes were down-regulated (Supplement Table S1). As shown in Fig. 2A and Fig. 2B, the distribution of differentially expressed genes was shown by heat map and volcano map. The structure of a-solanine was displayed in Fig. 2C. Then 399 target genes of a-solanine were obtained (Supplement Table S2). Further analysis revealed a total of 130 targets for a-solanine and NSCLC (Fig. 2D). The details of these 130 genes were shown in Supplement Table S3.

\section{Results Of Ppi Network Construction And Core Gene}

The candidate targets of a-solanine and NSCLC were introduced into the String website, and the network interaction diagram of them was obtained (Supplementary Figure S1). In the cytoscape interactive network of a-solanine and NSCLC there are 110 nodes and 712 edges. CytoNCA was used to obtain the core target network through DC, BC, CC, EC, NC and LAC parameters. In the core target network, a-solanine and NSCLC have 31 nodes and 395 edges, which mainly include GPI, ALDOA, TPI1, PKLR, LDHA and $\mathrm{ALDH}_{3}$, etc. (shown in Fig. 3).

\section{Results of GO and KEGG enrichment analysis targeting between a-solanine and NSCLC}

GO enrichment analysis was conducted on the common potential target gene of a-solanine and NSCLC. The top10 significantly enriched terms in BP, CC, and MF categories were shown in Fig. 4A. The biological processes are mainly purine nucleotide metabolic process, the cellular components mainly include the cytoplasmic vesicle lumen, and the molecular functions mainly include amide binding, etc. KEGG 
pathway analysis mainly included Glycolysis/Gluconeogenesis and Biosynthesis of amino acids signaling pathway in Fig. 4B.

\section{Experimental Validation}

\section{a-solanine inhibited the growth, migration and invasion of A549 and PC-9 cells.}

Various cell function experiments were first carried out in order to verify the effect of a-solanine on NSCLC. When the concentration of a-solanine was increased $(0,10,12,14,16,18,20,22,24 \mu \mathrm{g} / \mathrm{mL})$, the viability of A549 and PC-9 cells was decreased in a dose-dependent and time-dependent manner at different time points ( 24 h, 48 h) (Fig. 5A). The IC50 values of a-solanine on A549 and PC-9 cell lines were $18 \mu \mathrm{g} / \mathrm{mL}$, respectively. It can be seen from the Fig. 5B and Fig. 5C, a-solanine inhibited A549 and PC-9 cell migration and invasion in a dose-dependent manner. In brief, these data confirmed that a-solanine may inhibit the growth of NSCLC cells in vitro.

\section{a-solanine Induces Apoptosis Of A549 And Pc-9 Cells}

The purpose of this experiment was to prove that a-solanine inhibited NSCLC cell apoptosis. Annexin VFITC/PI double staining was used to detect the apoptotic cells. As can be seen from the Fig. 6, after $24 \mathrm{~h}$ of a-solanine treatment, the proportion of early and late apoptotic cells in the $12 \mu \mathrm{g} / \mathrm{mL}$ group was 10.51 $\pm 0.34 \%$ of PC-9. In the $18 \mu \mathrm{g} / \mathrm{mL}$ group, the early and late apoptotic cells accounted for $2.40 \pm 0.03 \%$ of A549 and $11.10 \pm 0.59 \%$ of PC-9, In $24 \mu \mathrm{g} / \mathrm{mL}$, the apoptosis rate of A549 was $11.91 \pm 0.93 \%$, and PC-9 was $15.67 \pm 1.42 \%$, both of which were significantly higher than those in $0 \mu \mathrm{g} / \mathrm{mL}$ group. The results displayed that a-solanine could promote the apoptosis of A549 and PC-9 cells.

\section{The Result Of Targeted Energy Metabolite Detection}

According to network pharmacological results, a-solanine may exert anti-tumor effect through energy metabolism pathway. To further verify the results, we carried out the detection of targeted energy metabolites. Firstly, the biological duplication among samples in the study group was investigated by means of sample correlation heat map (shown in Fig. 7A). The higher the correlation coefficient of intragroup samples relative to inter-group samples, the more reliable the differential metabolites obtained.

In this study, 65 energy metabolites were quantifiable (Supplementary Table S4 and Supplementary Figure S2). Based on the assessment of OSC partial least squares-discriminant analysis (OPLS-DA), the profile of metabolites of a-solanine group and control group could be clearly separated (shown in Fig. 7B). The S-plot of OPLS-DA showed the differential metabolites screened by variable importance in projection (VIP) value, with red dots representing VIP $>1$ and green dots representing VIP $<1$ (shown in Fig. 7C). Qualitative and quantitative analysis was conducted on the detected differential metabolites, and the metabolites with large difference in change times were shown, as shown in the bar chart of 
metabolites (Fig. 7D). Clustered heat maps reveal the total differences of differential metabolites (Fig. 7E). Differential metabolite fiddle plots also showed changes in metabolites in the administration and control groups (Fig. 7F). To further evaluate the relevance of metabolic pathways that are altered by a-solanine treatment, the KEGG pathway database was used to identify pathway enrichment patterns for the significantly altered metabolites. The results showed that differential metabolites were mainly involved in biosynthesis of amino acids related pathways (Fig. 7G).

\section{a-solanine Down-regulates Energy Metabolism Pathway Related Proteins Expression}

In order to preliminarily verify the effect of a-solanine on energy metabolism pathway, immunofluorescence technology was used to detect the changes of related proteins in the control group and the administration group, and it was found that the expression of GPI, ALDOA, TPI1, PKLR, LDHA and $\mathrm{ALDH}_{3}$ in the administration group was significantly reduced (Fig. 8). As shown in Fig. 9, A549 and PC-9 were treatment with a-solanine at $0,12,18$ and $24 \mu \mathrm{g} / \mathrm{mL}$ significantly inhibited the energy metabolism pathway related proteins. The western blot results were consistent with immunofluorescence. These results suggested that a-solanine inhibited the growth of NSCLC through the energy metabolism pathway.

\section{Discussion}

NSCLC is a heterogeneous tumor, accounting for approximately $85 \%$ of all newly diagnosed lung cancers [26]. Due to inadequate screening programs and late onset of clinical symptoms, most patients are already in the advanced stage of disease when they are diagnosed. Therefore, the prognosis of patients is poor [27]. With the deepening of understanding of tumor molecular biology, the treatment of NSCLC has gradually entered the era of precise treatment based on tumor molecular and histological characteristics. All this information makes possible the development of individualized molecular targeted therapy ${ }^{[28]}$. asolanine is the main component of Solanum, which has a variety of pharmacological effects. In this study, the mechanism of a-solanine against NSCLC was studied by integrating network pharmacology and molecular biology techniques.

In drug discovery, methods based on network pharmacology have given us a deeper understanding of multi-layer drug information, which can provide support for the development of rational drug design and a better understanding of the mechanism of drug multiple actions ${ }^{[29]}$. For example, through network pharmacological analysis, it was found that the possible mechanisms of triptolide include regulation of immune and inflammatory responses, promotion of cell apoptosis and inhibition of tumor development [30]. Artemisia argyi may produce gastric mucosal protection through coordinated regulation of NF-kB, HIF-1, TNF, VEGF, Toll-like receptor signaling pathways and other biological pathways ${ }^{[31]}$. Using the method of network pharmacology, the mechanism of Tripterygium wilfordii in the treatment of acute myeloid leukemia is explained in detail, that is, the main pathways of treatment targets are Toll-like 
receptor signaling pathway, NF-KB signaling pathway and HIF-1 signaling pathway ${ }^{[32]}$. In this study, first download the target of a-solanine from the PharmMapper database and the target of the treatment of NSCLC from the TCGA database, then use R software to perform differential analysis of different expressions to obtain candidate genes for a-solanine to act on NSCLC. Use Cytoscape software to construct a network diagram of drugs and diseases, and establish a multi-level "drug target-disease" network to reveal the relationship between a-solanine and NSCLC. Finally, GO analysis is conducted through candidate genes. From three aspects, the biological process is mainly involved in purine nucleotide metabolism. In terms of cell components, it is mainly related to the cytoplasmic vesicle cavity, and it is inseparable from the combination of amide in molecular function. At the same time, KEGG analysis found that the effect of a-solanine against NSCLC was mainly related to the metabolic pathway, especially the glycolytic pathway, indicating that a-solanine may produce anti-tumor effects through the energy metabolism pathway. It has been suggested that the occurrence, development and metastasis of cancer are highly regulated by transcription factors, and transcription regulators promote the regulation of energy metabolism pathways, which mainly depend on the tumor microenvironment ${ }^{[33]}$. The analysis found that rapamycin plays a key role in the connection between glycosphingolipids and glutamine metabolism and mitochondrial signaling ${ }^{[34]}$.

According to the experimental results, it can be concluded that A549 and PC-9 were inhibited with the increase of the administration concentration and the extension of the administration time, showing a concentration and time dependence. Finally, it was determined that the administration concentration of A549 and PC-9 was IC50, which was about $18 \mu \mathrm{g} / \mathrm{mL}$. Further studies have shown that a-solanine inhibits the invasion and migration of $A 549$ and PC-9, and increases with the increase of drug concentration. asolanine induces apoptosis of A549 and PC-9, and the apoptotic rate increases with the increase of drug concentration. Previous studies have shown that the induction of cancer cell apoptosis by steroid alkaloids is one of the reasons for its anti-cancer effect ${ }^{[8,35,36]}$. This may also be an important reason why a-solanine exhibits anti- NSCLC effects.

According to the results of network pharmacology analysis, a-solanine may produce anti-tumor effects through metabolic pathways, especially energy metabolism. This study further detected the expression of energy metabolites through targeted metabolomics. At present, small molecule metabolites play an important role in cancer screening, diagnosis and prognosis ${ }^{[37]}$. It is also a good method for evaluating drug efficacy and mechanism of action ${ }^{[38]}$. The results of metabolomics experiments showed that the Administration group compared with the control group, the small molecule metabolites related to amino acid biosynthesis had significant changes. We focused on the first 20 metabolites (Fig. 7D and Fig. 7F) with significant changes. Among them, 15 small-molecule metabolites increased in synthesis and the other 5 decreased. Combined with the KEGG pathway diagram and experiments, it can be obtained that asolanine affects the synthesis and elimination of small molecule metabolites by inhibiting amino acid synthase and metabolic enzymes, that is, inhibiting synthase. The decrease in the synthesis of amino acids shows a decrease in the number of amino acids; on the contrary, the inhibition of amino acid 
metabolism enzymes reduces the elimination of amino acid metabolism and accumulates, which shows an increase in the number of amino acids.

In order to further verify that a-solanine may act on NSCLC through glycolysis pathway, related proteins such as GPI, ALDOA, TPI1, PKLR, LDHA and $\mathrm{ALDH}_{3}$ in glycolysis pathway were studied. This is mutually confirmed with the core target in network pharmacology. In the immunofluorescence experiment, it can be seen that the expression of the above proteins which treated with a-solanine reduced. The western blot experiment was consistent with the immunofluorescence result. The above two experiments showed that the expression of GPI, ALDOA, TPI1, PKLR, LDHA and ALDH 3 decreased after A549 and PC-9 were treated with a-solanine, indicating the mechanism of a-solanine is related to these proteins and is consistent with the results of the research of network pharmacology.

The results showed that a-solanine may achieve the purpose of anti-tumor by affecting energy metabolism. Adequate amino acid supply is important for cancer to maintain its proliferation power. In addition to its direct role as a substrate for protein synthesis, it can also play a role in energy production, driving nucleoside synthesis and maintaining cell redox homeostasis ${ }^{[39]}$. At present, many differences in the metabolism of specific amino acids in prostate cancer have been found. Other key requirements of the amino acid library come from serine, single carbon cycle, glycine synthesis pathway and folate metabolism, which are the main intermediates for the synthesis of nucleobases necessary for rapidly proliferating cells ${ }^{[40]}$. The currently known metabolic changes related to cancer can be roughly divided into six aspects ${ }^{[41]}$ : (1) Glucose and amino acid intake disorders (2) Random access to nutrients (3) Biosynthesis using glycolysis/TCA cycle intermediates (4) Increased demand for nitrogen (5) Metabolitedriven gene regulation changes (6) Metabolism interaction with the microenvironment. Generally speaking, tumor cells metabolize glucose, lactic acid, pyruvate, hydroxybutyrate, acetic acid, and glutamine much faster than non-tumor cells ${ }^{[42]}$. Studies have pointed out that amino acid metabolism starvation therapy has become essential in the treatment of many tumors, and non-essential amino acids are particularly prominent ${ }^{[43]}$. In energy metabolism, glucose undergoes glycolysis in the cytoplasm to produce phosphoglucose and phosphoglycerate. These two substances can produce histidine, serine, glycine, and cysteine. Phosphoglycerate can be further metabolized to produce pyruvate, which can be converted into alanine, valine, and leucine. Then, if pyruvate enters the mitochondria and undergoes further oxidation, that is, enters the tricarboxylic acid cycle, a-ketoglutarate can be produced. $a-$ ketoglutarate can provide backbone atoms for glutamate amino acids, which can be converted into glutamic acid, glutamine, and further into ornithine, citrulline, arginine, proline, and hydroxyproline acid. If ketoglutarate continues to be converted in the tricarboxylic acid cycle, it can be converted to oxaloacetic acid. Oxaloacetate can be converted into aspartic acid, asparagine, lysine, threonine, methionine, and isoleucine. In addition to glycolysis of glucose in the cytoplasm to form pyruvate, the pentose phosphate pathway can also produce tryptophan, phenylalanine, and tyrosine. Compared with normal cells, tumor cells need a lot of amino acids to synthesize their own substances in order to achieve rapid growth, invasion and migration ${ }^{[44]}$. By enhancing aerobic glycolysis, cancer cells provide more carbon intermediates for the synthesis of nucleotides, amino acids and lipids, and promote their anabolism. This 
experiment found through metabolomics that more than 10 amino acids including alanine, cysteine, serine, tyrosine, etc., increased after a certain concentration of a-solanine, and these amino acid metabolites and energy were significantly changed. The metabolic pathways are closely related. Among them, alanine, cysteine, serine and threonine are related to the energy metabolism intermediate product pyruvate, while asparagine, tyrosine, glutamine and arginine are respectively related to oxaloacetate in the citric acid cycle, Fumaric acid and a-ketoglutarate are related. Combining with the previous discussion and the results of metabolomics, it is found that the intermediate product of glycolysis pathway glyceraldehyde 3-phosphate rises, which can indicate that the expression of energy metabolism enzymes inhibited by a-solanine can cause disorder of its metabolic intermediates and interfere with cancer. Cell energy metabolism inhibits tumor cell proliferation, migration and invasion, and promotes apoptosis. The schematic diagram of the inhibition mechanism of a-solanine on NSCLC (Fig. 10).

\section{Conclusion}

In conclusion, the pharmacological mechanism of a-solanine inhibiting NSCLC was discussed by combining network pharmacological prediction and experimental verification. a-solanine may inhibit the proliferation and survival of NSCLC by regulating glycolysis related pathways. This study further suggests that the combination of network pharmacological prediction and experimental verification can provide a useful tool for the detailed description of the mechanism of action of TCM. The potential therapeutic effect of a-solanine on NSCLC may benefit from further clinical trials in patients with lung cancer.

\section{Declarations}

\section{DATA AVAILABILITY STATEMENT}

The raw data supporting the conclusions of this manuscript will be made available by the authors, without undue reservation, to any qualified researcher.

\section{ETHICS STATEMENT}

No humans and animals were involved in the study.

\section{AUTHOR CONTRIBUTIONS}

ZGW and ZGX contributed to the design of the study. TZ, YKS and YFH performed the experiments and prepared the manuscript. TZ, JSW, JXL and LQY analyzed the data. GWZ, TZ, YSZ, JS and MXL interpreted results and prepared the manuscript. XW, FKD, YC, MJC and YY interpreted the results and revised manuscript. The final version of the manuscript has been read and approved by all authors.

\section{FUNDING}


This work was supported by the Project of Science and Technology Department of Sichuan Province (No. 2021YJ0445), the Project of Doctoral Research Initiation Foundation of Affiliated Hospital of Southwest Medical University (No. 18104), the Joint project of Luzhou Traditional Chinese Medicine Hospital and Southwest Medical University (No.2018-LH004), the Collaborative Project of Luzhou Government and Southwest Medical University (2018LZXNYD-PT02), and the Project of Luzhou Science and Technology Bureau (No.2019-81).

\section{COMPETING INTEREST}

The authors declare that they have no competing interests.

\section{ACKNOWLEDGEMENTS}

We would like to thank the Wuhan Metware Biotechnology Co., Ltd. (Wuhan, China) for quantification of targeting energy metabolites.

\section{References}

1. Henley, S.J., et al., Annual report to the nation on the status of cancer, part I: National cancer statistics. Cancer, 2020. 126(10): p. 2225-2249.

2. Judd, J. and H. Borghaei, Combining Immunotherapy and Chemotherapy for Non-Small Cell Lung Cancer. Thorac Surg Clin, 2020. 30(2): p. 199-206.

3. Duma, N., R. Santana-Davila, and J.R. Molina, Non-Small Cell Lung Cancer: Epidemiology, Screening, Diagnosis, and Treatment. Mayo Clin Proc, 2019. 94(8): p. 1623-1640.

4. Shin, J.Y., J.K. Yoon, and G. Marwaha, Progress in the Treatment and Outcomes for Early-Stage NonSmall Cell Lung Cancer. Lung, 2018. 196(3): p. 351-358.

5. Abdel Karim, N. and K. Kelly, Role of Targeted Therapy and Immune Checkpoint Blockers in Advanced Non-Small Cell Lung Cancer: A Review. Oncologist, 2019. 24(9): p. 1270-1284.

6. Giraud, N., S. Abdiche, and R. Trouette, Stereotactic radiotherapy in targeted therapy treated oligometastatic oncogene-addicted (non-small-cell) lung cancer. Cancer Radiother, 2019. 23(4): p. 346354.

7. Gebhardt, C., The historical role of species from the Solanaceae plant family in genetic research. Theor Appl Genet, 2016. 129(12): p. 2281-2294.

8. Yan, X., et al., a-Solanine inhibits growth and metastatic potential of human colorectal cancer cells. Oncol Rep, 2020. 43(5): p. 1387-1396.

9. Friedman, M., Chemistry and anticarcinogenic mechanisms of glycoalkaloids produced by eggplants, potatoes, and tomatoes. J Agric Food Chem, 2015. 63(13): p. 3323-37.

10. Sun, H., et al., Solanine induces mitochondria-mediated apoptosis in human pancreatic cancer cells. Biomed Res Int, 2014. 2014: p. 805926. 
11. Mohsenikia, M., et al., Therapeutic effects of dendrosomal solanine on a metastatic breast tumor. Life Sci, 2016. 148: p. 260-7.

12. Lv, C., et al., Antitumor efficacy of a-solanine against pancreatic cancer in vitro and in vivo. PLoS One, 2014. 9(2): p. e87868.

13. Wang, Y., et al., a-Solanine Modulates the Radiosensitivity of Esophageal Cancer Cells by Inducing MicroRNA 138 Expression. Cell Physiol Biochem, 2016. 39(3): p. 996-1010.

14. Ji, Y.B., et al., Induction of apoptosis in HepG2 cells by solanine and Bcl-2 protein. J Ethnopharmacol, 2008. 115(2): p. 194-202.

15. Lu, M.K., et al., a-Solanine inhibits human melanoma cell migration and invasion by reducing matrix metalloproteinase-2/9 activities. Biol Pharm Bull, 2010. 33(10): p. 1685-91.

16. Amankwatia, E.B., et al., MicroRNA-224 is associated with colorectal cancer progression and response to 5-fluorouracil-based chemotherapy by KRAS-dependent and-independent mechanisms. Br J Cancer, 2015. 112(9): p. 1480-90.

17. Asuthkar, S., et al., Epigenetic regulation of miRNA-211 by MMP-9 governs glioma cell apoptosis, chemosensitivity and radiosensitivity. Oncotarget, 2012. 3(11): p. 1439-54.

18. Shin, J.S., et al., a-Solanine Isolated From Solanum Tuberosum L. cv Jayoung Abrogates LPSInduced Inflammatory Responses Via NF-KB Inactivation in RAW 264.7 Macrophages and EndotoxinInduced Shock Model in Mice. J Cell Biochem, 2016. 117(10): p. 2327-39.

19. Zhang, F., et al., Anticancer function of a-solanine in lung adenocarcinoma cells by inducing microRNA-138 expression. Tumour Biol, 2016. 37(5): p. 6437-46.

20. Lin, L.T., C.Y. Choong, and C.J. Tai, Solanine Attenuated Hepatocarcinoma Migration and Invasion Induced by Acetylcholine. Integr Cancer Ther, 2020. 19: p. 1534735420909895.

21. Friedman, J.R., et al., Acetylcholine signaling system in progression of lung cancers. Pharmacol Ther, 2019. 194: p. 222-254.

22. Hopkins, A.L., Network pharmacology: the next paradigm in drug discovery. Nat Chem Biol, 2008. 4(11): p. 682-90.

23. Wang, N., et al., Network pharmacology-based analysis on bioactive anti-diabetic compounds in Potentilla discolor bunge. J Ethnopharmacol, 2019. 241: p. 111905.

24. Kibble, M., et al., Network pharmacology applications to map the unexplored target space and therapeutic potential of natural products. Nat Prod Rep, 2015. 32(8): p. 1249-66.

25. Yuan, M., et al., Ex vivo and in vivo stable isotope labelling of central carbon metabolism and related pathways with analysis by LC-MS/MS. Nat Protoc, 2019. 14(2): p. 313-330.

26. Gelatti, A.C.Z., A. Drilon, and F.C. Santini, Optimizing the sequencing of tyrosine kinase inhibitors (TKIS) in epidermal growth factor receptor (EGFR) mutation-positive non-small cell lung cancer (NSCLC). Lung Cancer, 2019. 137: p. 113-122.

27. Gridelli, C., et al., Non-small-cell lung cancer. Nat Rev Dis Primers, 2015. 1: p. 15009. 
28. Ferrara, M.G., et al., Oncogene-Addicted Non-Small-Cell Lung Cancer: Treatment Opportunities and Future Perspectives. Cancers (Basel), 2020. 12(5).

29. Boezio, B., et al., Network-based Approaches in Pharmacology. Mol Inform, 2017. 36(10).

30. Zou, T., et al., Study on the Anti-Tumor Mechanism and Target of Triptolide based on Network Pharmacology and Molecular Docking. Recent Pat Anticancer Drug Discov, 2021.

31. Wang, Y., et al., Virtual screening of active compounds from Artemisia argyi and potential targets against gastric ulcer based on Network pharmacology. Bioorg Chem, 2019. 88: p. 102924.

32. Fang, T., L. Liu, and W. Liu, Network pharmacology-based strategy for predicting therapy targets of Tripterygium wilfordii on acute myeloid leukemia. Medicine (Baltimore), 2020. 99(50): p. e23546.

33. Rodríguez-Enríquez, S., et al., Transcriptional Regulation of Energy Metabolism in Cancer Cells. Cells, 2019. 8(10).

34. Schömel, N., G. Geisslinger, and M.S. Wegner, Influence of glycosphingolipids on cancer cell energy metabolism. Prog Lipid Res, 2020. 79: p. 101050.

35. Zheng, Y., et al., Solanine inhibits proliferation and promotes apoptosis of the human leukemia cells by targeting the miR-16/Bcl-2 axis. J buon, 2020. 25(3): p. 1614-1618.

36. El-Daly, S.M., et al., Synergistic Effect of a-Solanine and Cisplatin Induces Apoptosis and Enhances Cell Cycle Arrest in Human Hepatocellular Carcinoma Cells. Anticancer Agents Med Chem, 2019. 19(18): p. 2197-2210.

37. Seijo, L.M., et al., Biomarkers in Lung Cancer Screening: Achievements, Promises, and Challenges. J Thorac Oncol, 2019. 14(3): p. 343-357.

38. Armitage, E.G. and M. Ciborowski, Applications of Metabolomics in Cancer Studies. Adv Exp Med Biol, 2017. 965: p. 209-234.

39. Vettore, L., R.L. Westbrook, and D.A. Tennant, New aspects of amino acid metabolism in cancer. Br J Cancer, 2020. 122(2): p. 150-156.

40. Strmiska, V., et al., Prostate cancer-specific hallmarks of amino acids metabolism: Towards a paradigm of precision medicine. Biochim Biophys Acta Rev Cancer, 2019. 1871(2): p. 248-258.

41. Pavlova, N.N. and C.B. Thompson, The Emerging Hallmarks of Cancer Metabolism. Cell Metab, 2016. 23(1): p. 27-47.

42. Martinez-Outschoorn, U.E., et al., Cancer metabolism: a therapeutic perspective. Nat Rev Clin Oncol, 2017. 14(1): p. 11-31.

43. Geck, R.C. and A. Toker, Nonessential amino acid metabolism in breast cancer. Adv Biol Regul, 2016. 62: p. 11-17.

44. Tabe, Y., P.L. Lorenzi, and M. Konopleva, Amino acid metabolism in hematologic malignancies and the era of targeted therapy. Blood, 2019. 134(13): p. 1014-1023.

\section{Figures}




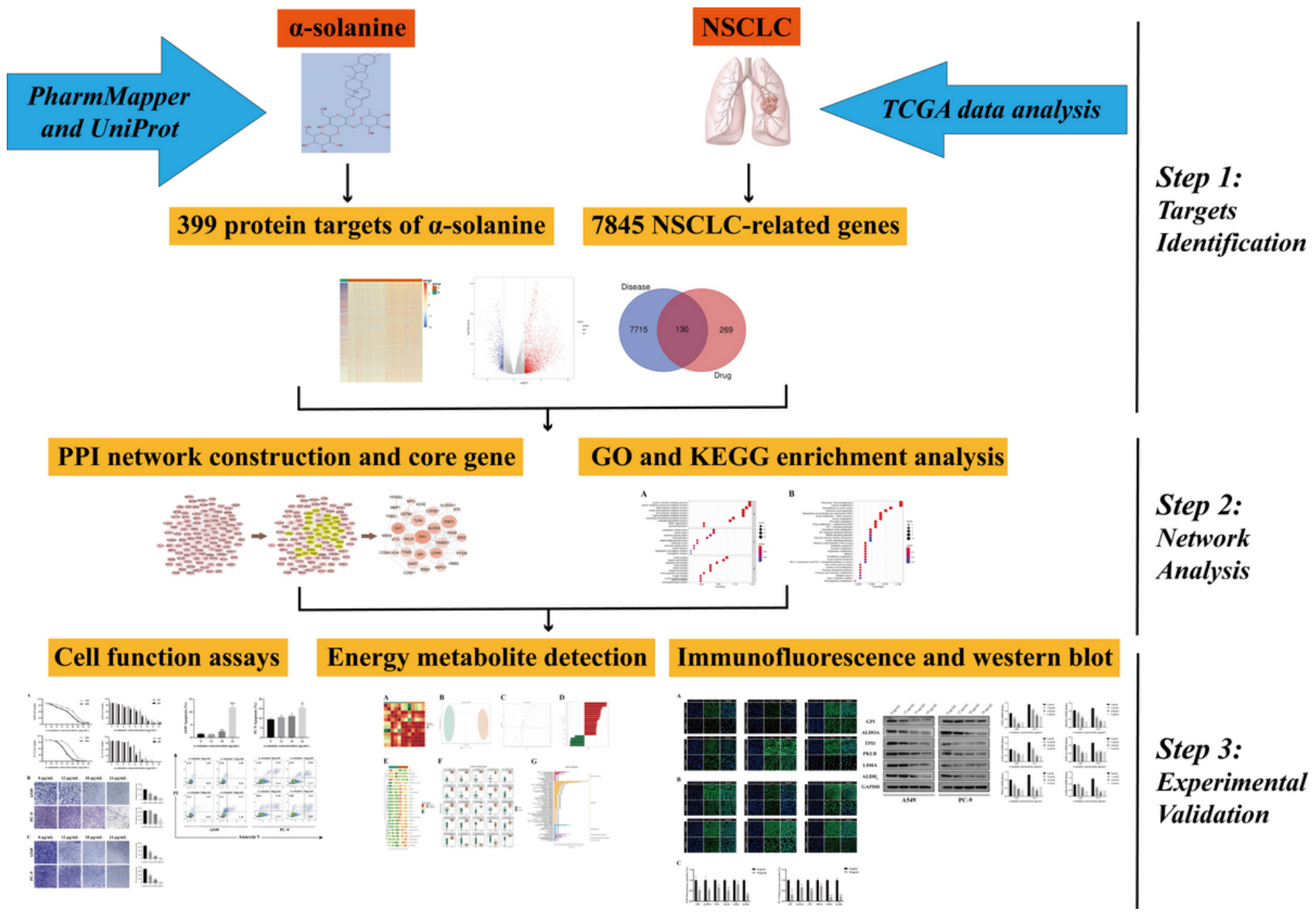

Figure 1

The strategic process of the current study. 
A

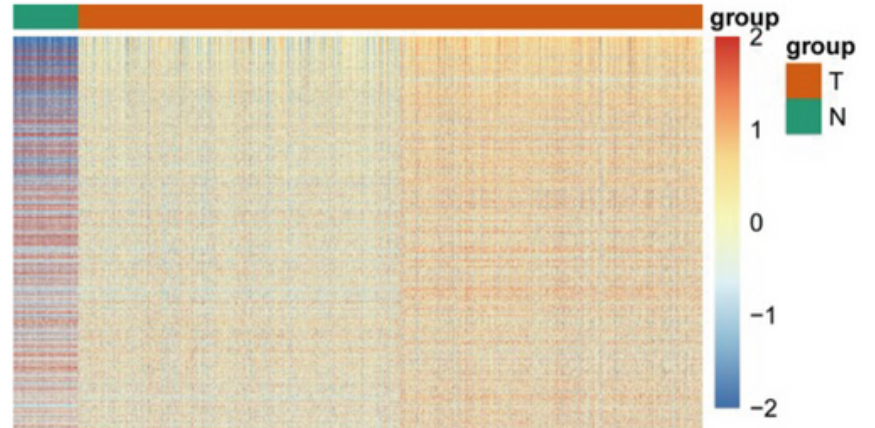

C

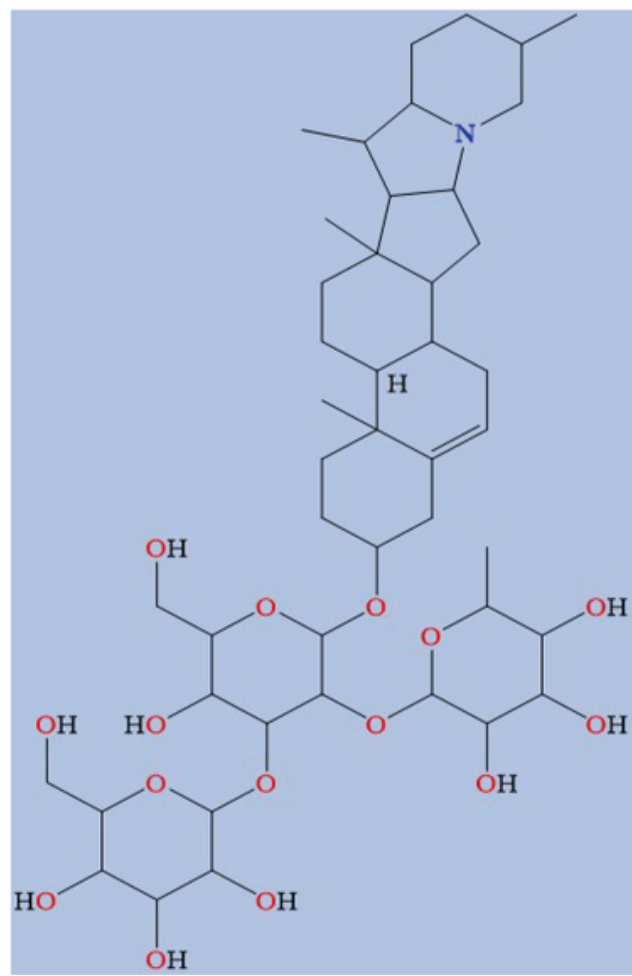

B

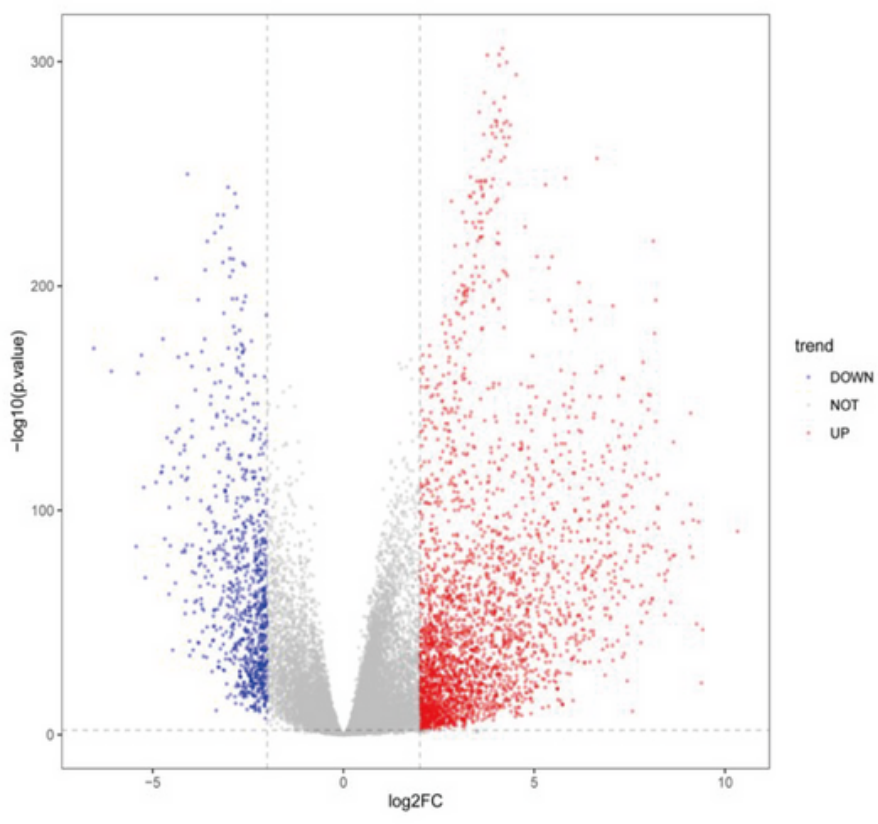

D

\section{Disease}

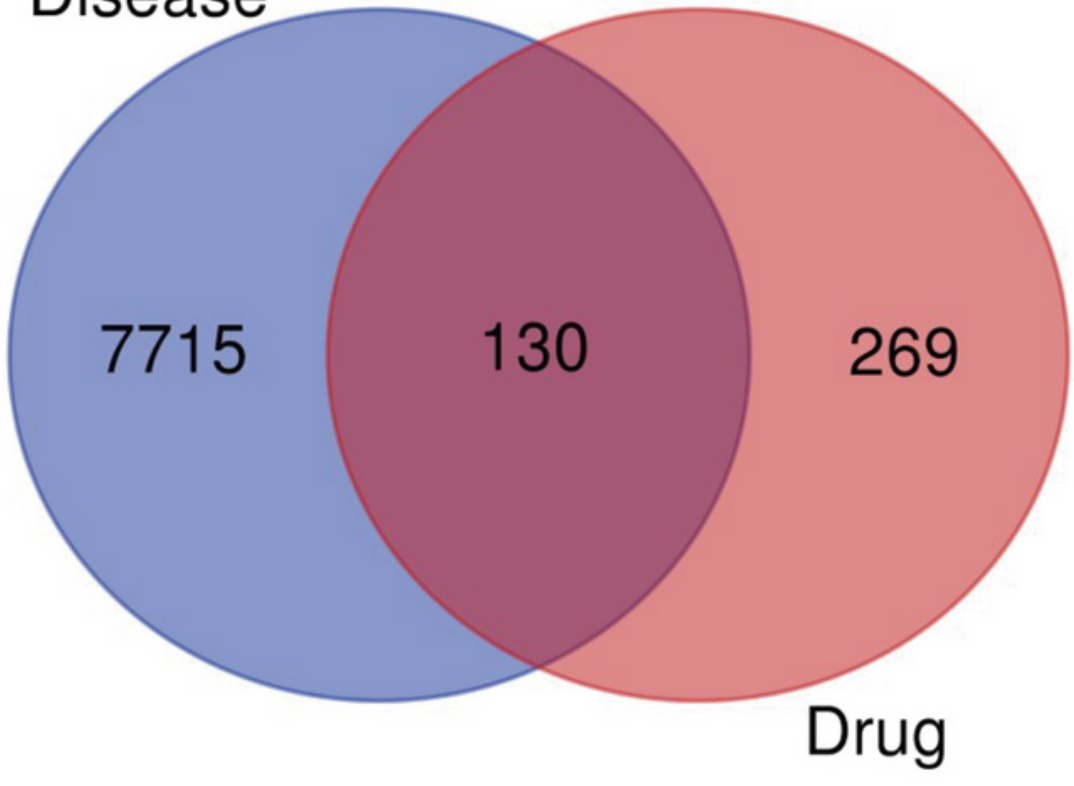

Figure 2

(A) Heat maps of different genes between NSCLC patients and normal samples.(B) Volcano maps of differential genes.(C) a-solanine structure diagram.(D)Venn plots of NSCLC targets and a-solanine targets. 


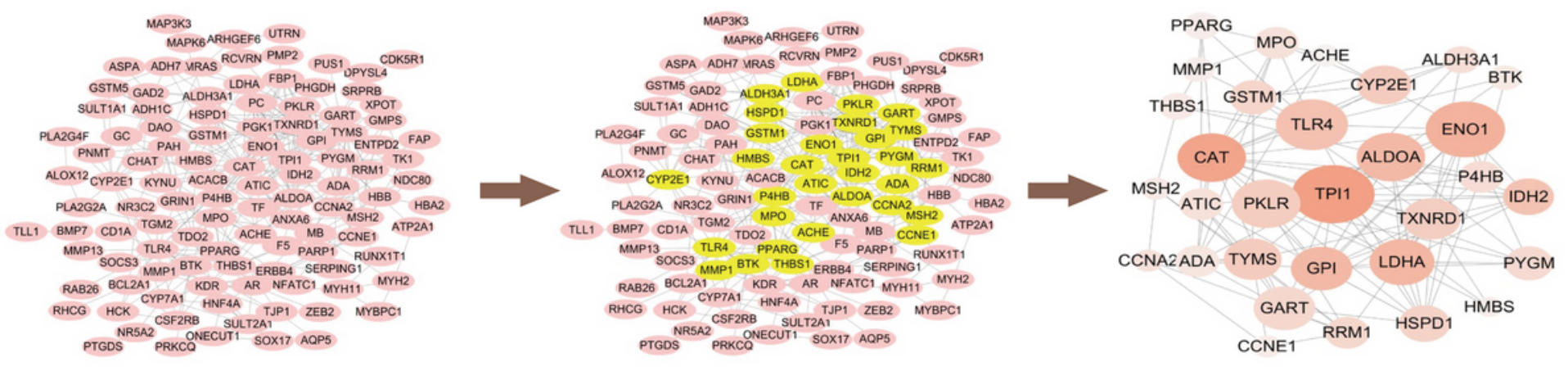

Figure 3

Process of topological screening for the PPI network. The yellow parts are the targets screened by calculation of $D C, B C, C C, E C, N C$ and LAC parameters. The last network diagram represents the core gene network. The more important targets were regarded as the targets with the bigger ellipse and darker color.

A

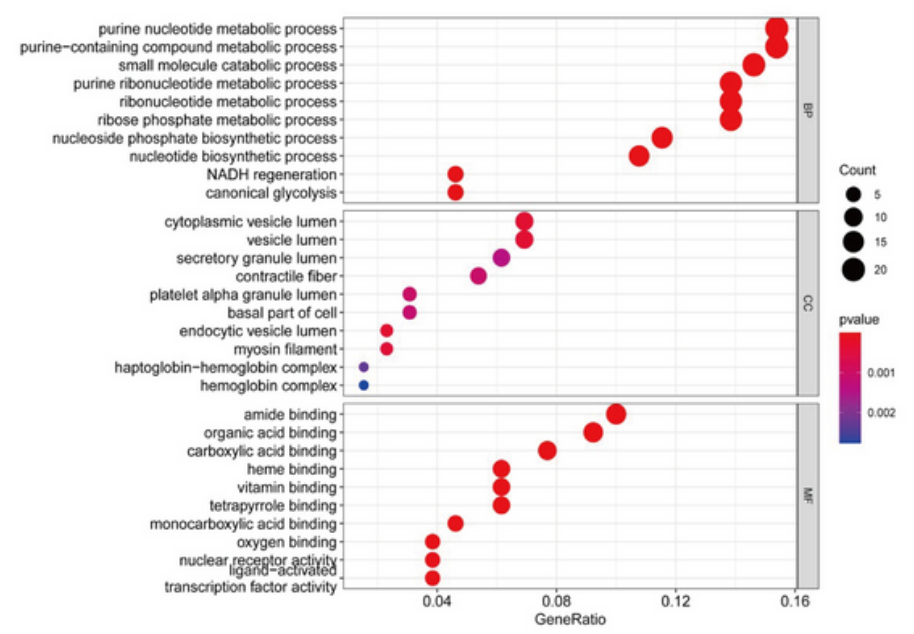

\section{B}

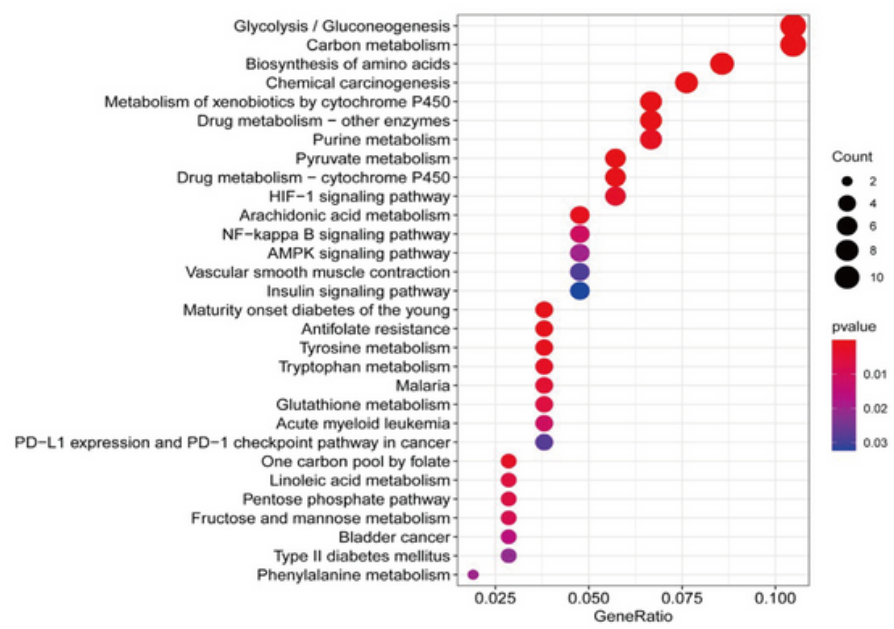

\section{Figure 4}

(A) GO enrichment analysis of the potential targets.BP: biological process; CC: cellular component; MF: molecular function. (B) KEGG pathways analysis of potential targets. 
A
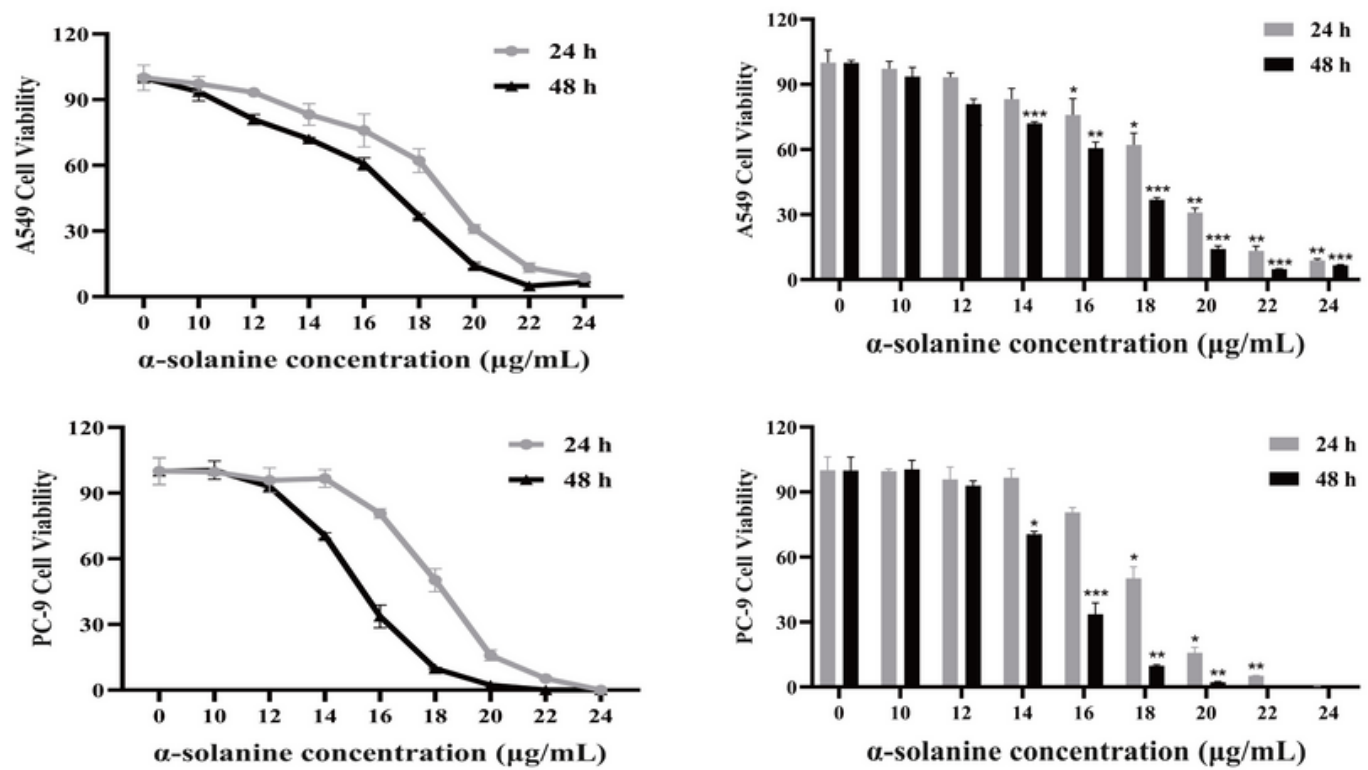

\section{В $\quad 0 \mu \mathrm{g} / \mathrm{mL} \quad 12 \mu \mathrm{g} / \mathrm{mL} \quad 18 \mu \mathrm{g} / \mathrm{mL} \quad 24 \mu \mathrm{g} / \mathrm{mL}$}
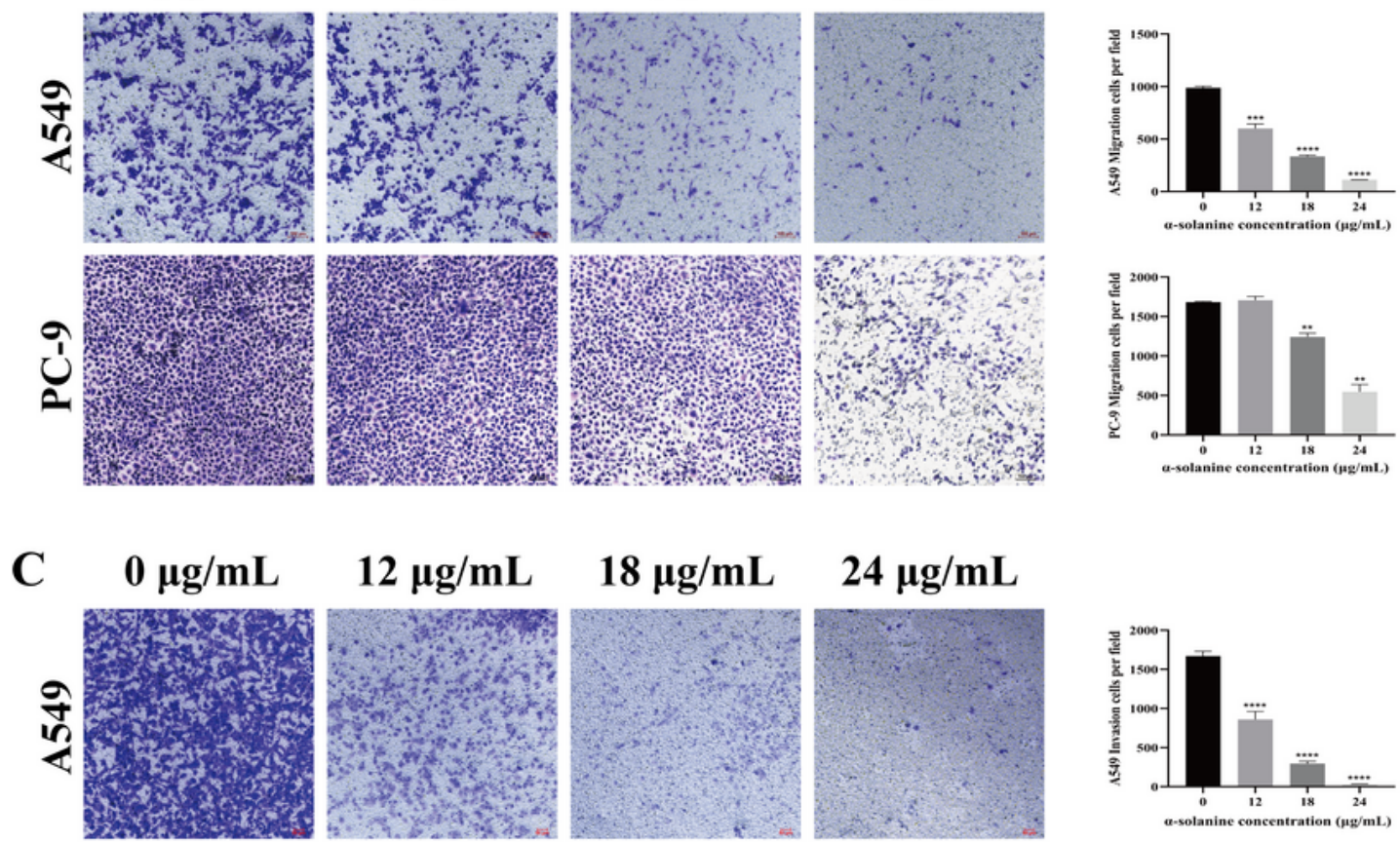

$12 \mu \mathrm{g} / \mathrm{mL}$

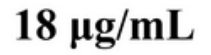

$24 \mu \mathrm{g} / \mathrm{mL}$
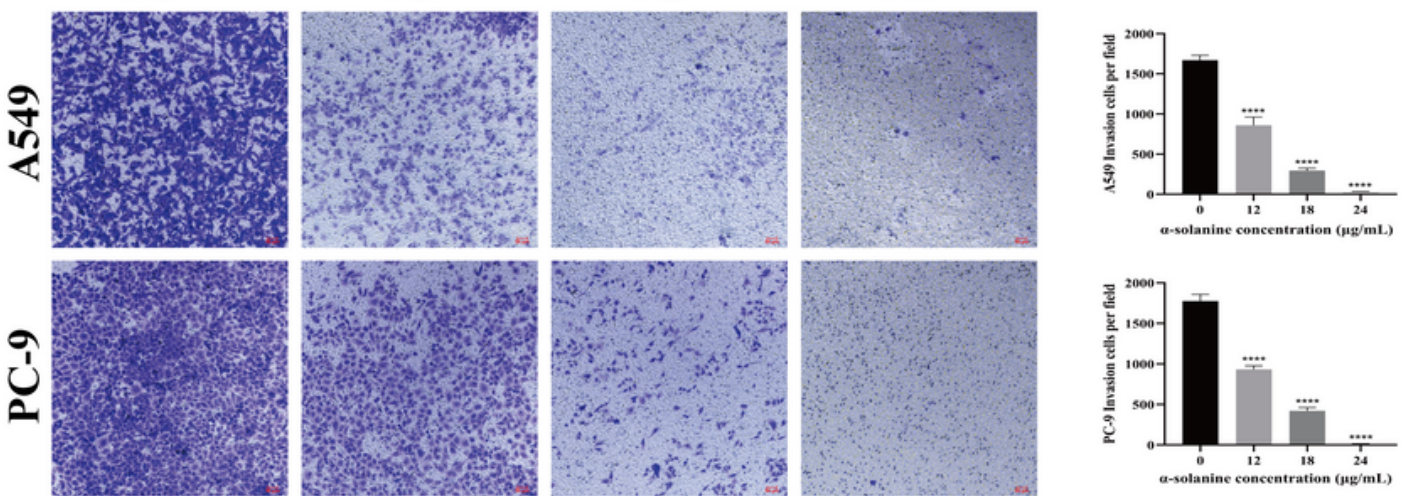

\section{Figure 5}

a-solanine inhibited NSCLC cell growth in Vitro. (A) Time- and dose-dependent effects of a-solanine treatment on the viability of A549 and PC-9 cells. (B) The representative images and statistical graphs of cell migration assay of A549 and PC- 9 cells. (C) The representative images and statistical graphs of cell

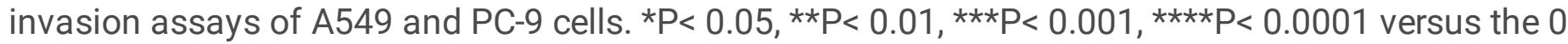
$\mu \mathrm{g} / \mathrm{mL}$ a-solanine group. 

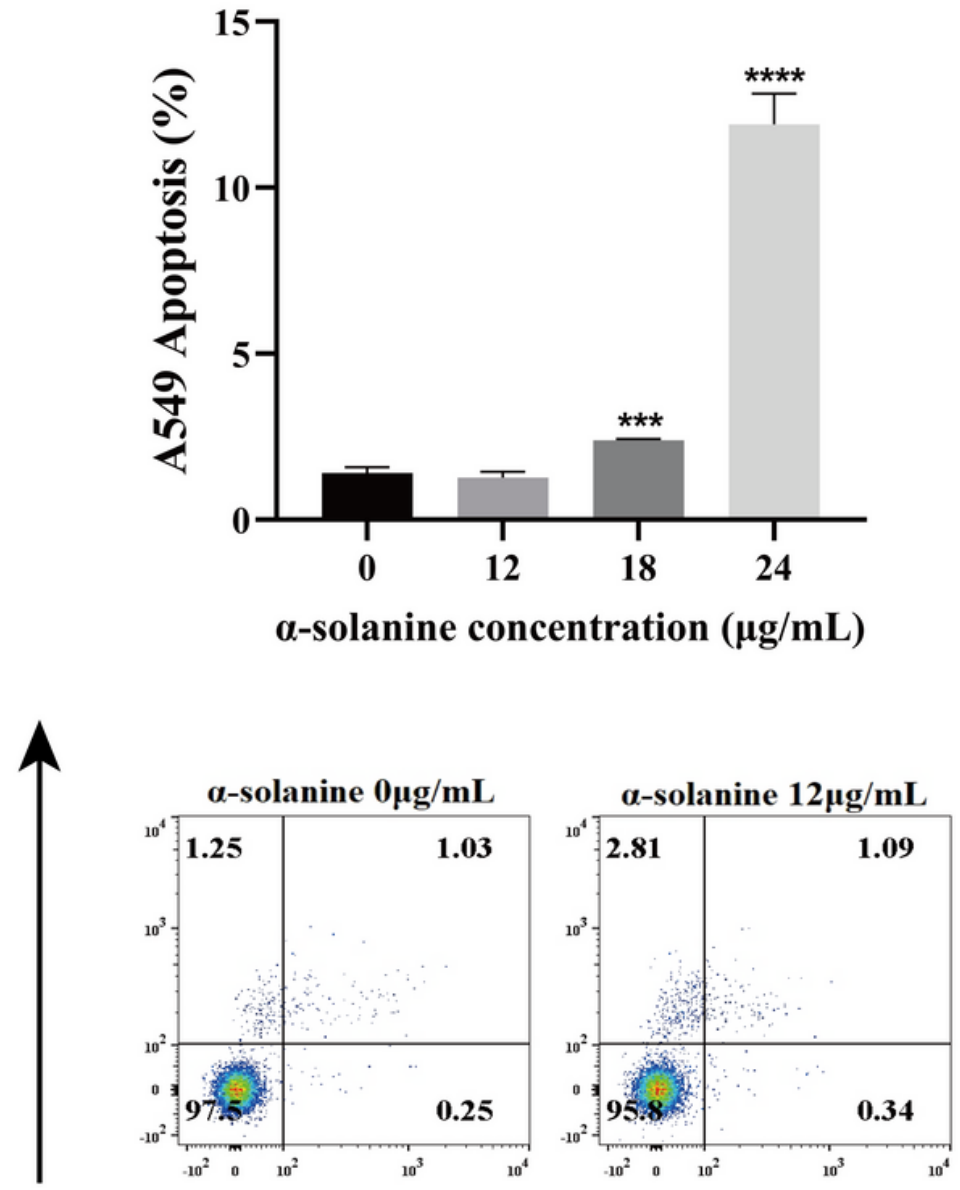

PI
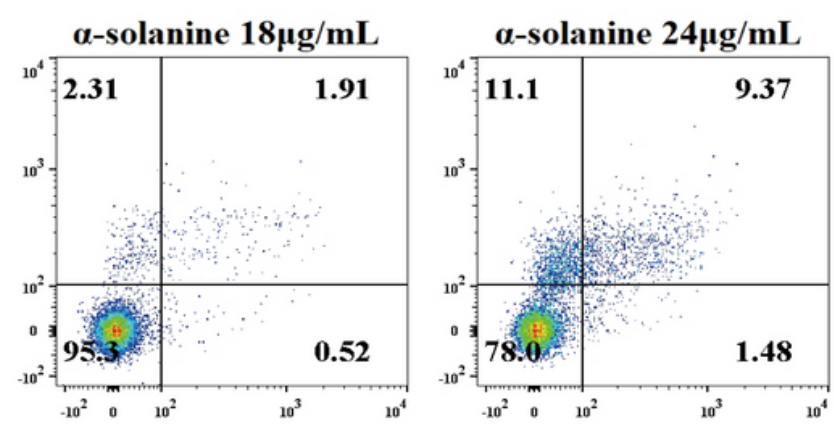

A549
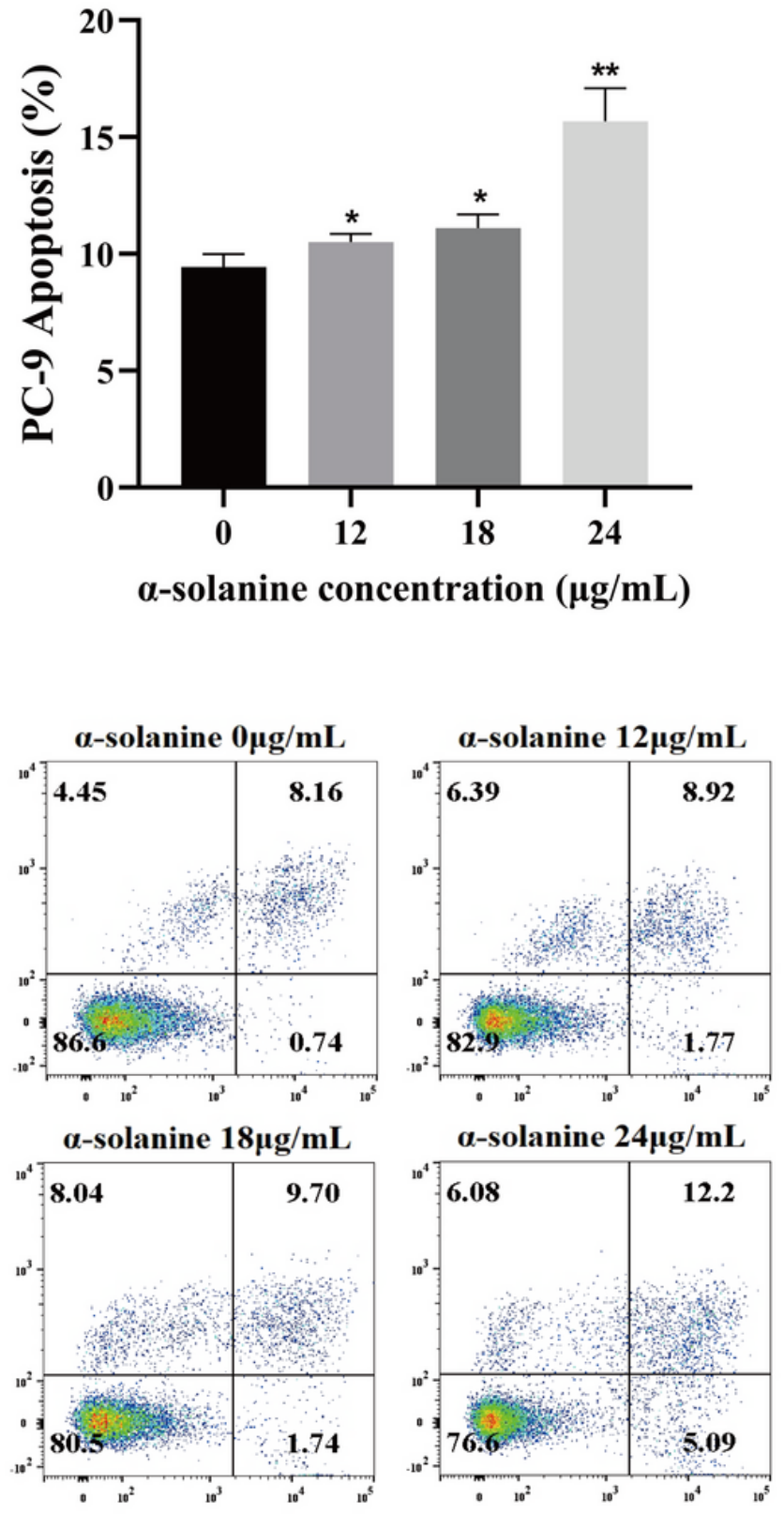

PC-9

\section{Annexin V}

Figure 6

a-solanine promoted NSCLC cell apoptosis in Vitro. Cell apoptosis measured by Annexin V-FITC/PI staining in A549 and PC-9 cells. ${ }^{*} P<0.05$, ${ }^{\star *} P<0.01$, ${ }^{\star \star \star} P<0.001$, ${ }^{\star \star \star *} P<0.0001$ versus the $0 \mu \mathrm{g} / \mathrm{mL}$ asolanine group. 

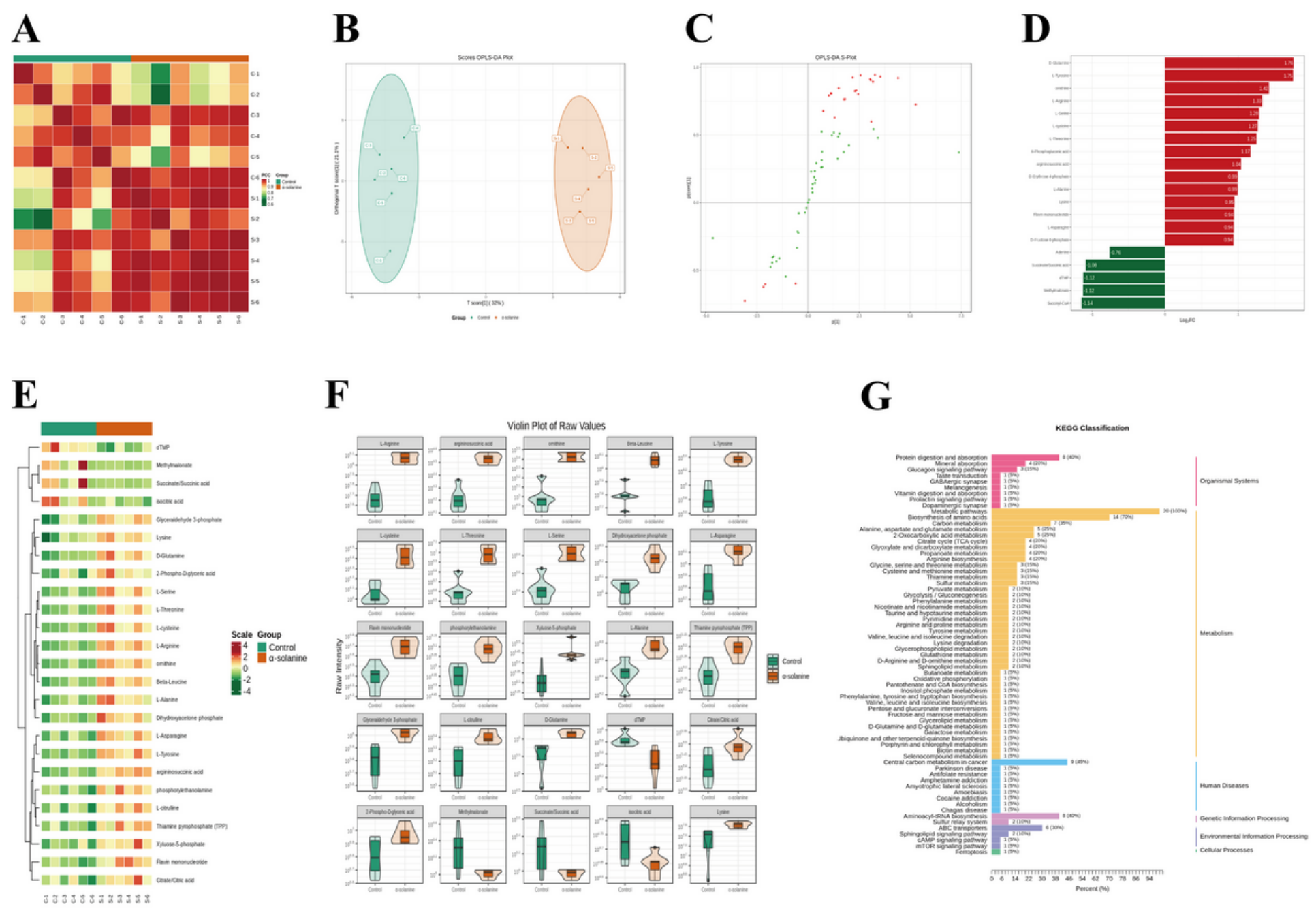

F

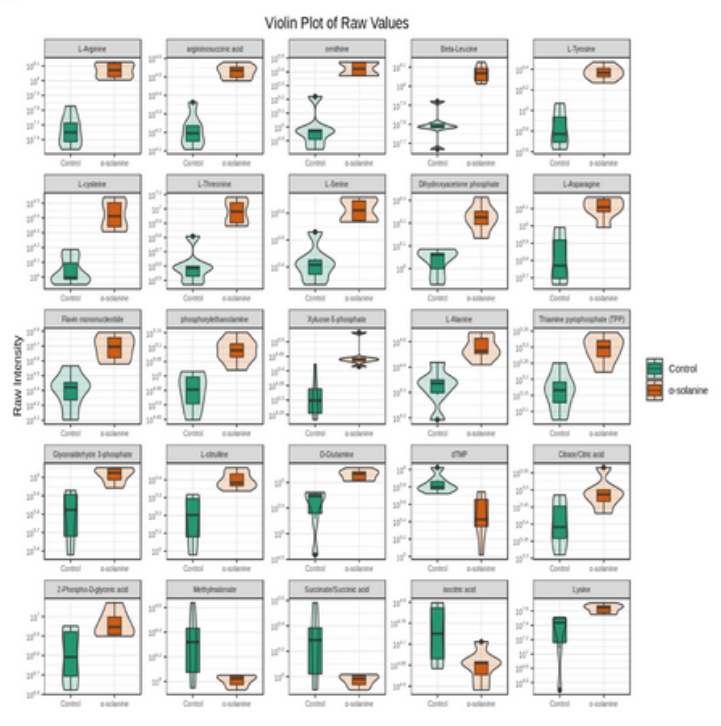

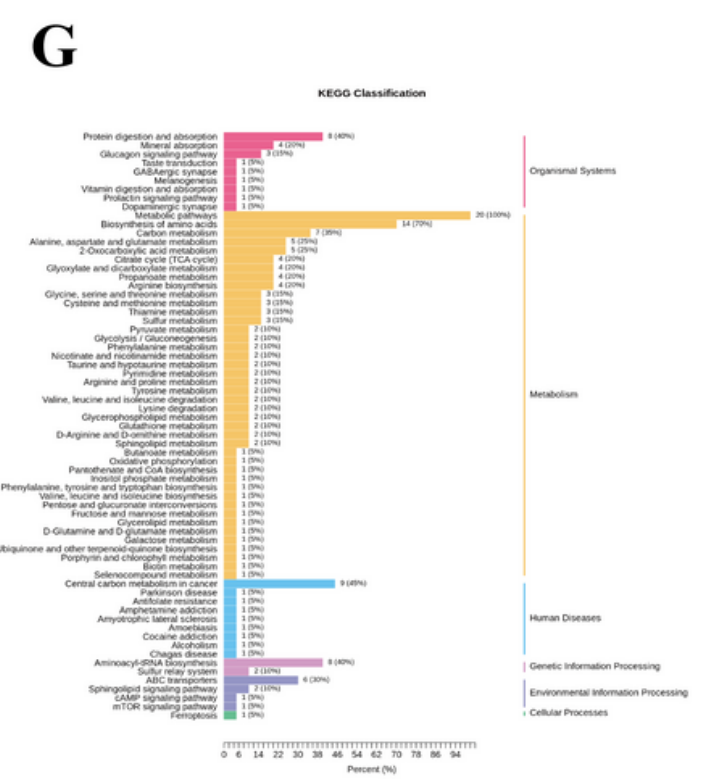

\section{Figure 7}

Comparison of energy metabolism profiles between control group and administration group. (A) Intersample correlation diagram. Horizontal and vertical coordinates represent the corresponding sample names, and color represents the value of phase relation. (B) OPLS-DA score chart for metabolomic data of the two groups. (C)Opls-da s-plot. The abscissa represents the cocorrelation coefficient between principal components and metabolites, while the ordinate represents the correlation coefficient between principal components and metabolites. (D) Histogram of differential multiple of metabolites. (E) Clustering heat map of differential metabolites, red represents high metabolic content and green represents low content. (F) Violin diagrams of differential metabolites. The abscissa is the sample and the ordinate is the expression quantity. (G) KEGG classification of differential metabolites. The ordinate is the name of KEGG metabolic pathway. The abscissa is the number of metabolites annotated to the pathway and its ratio to the total number of metabolites annotated. 
A
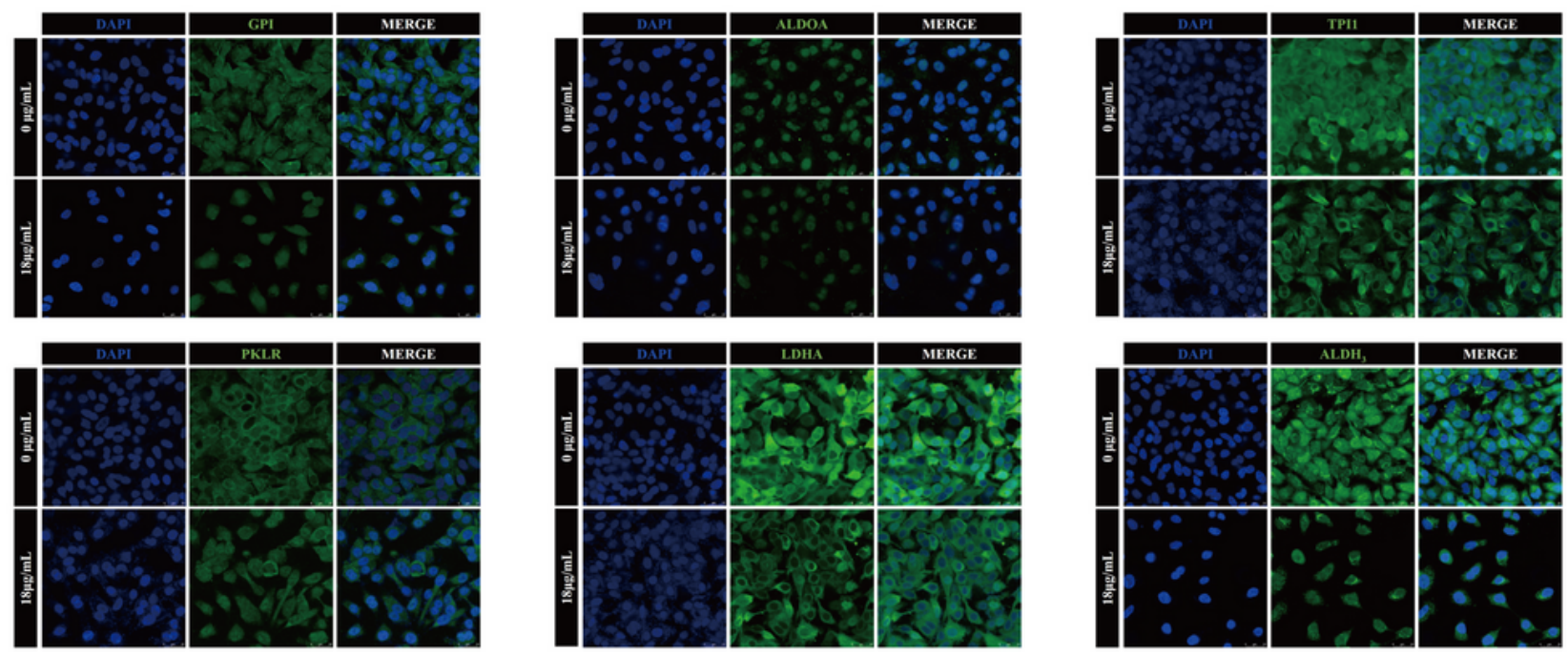

B
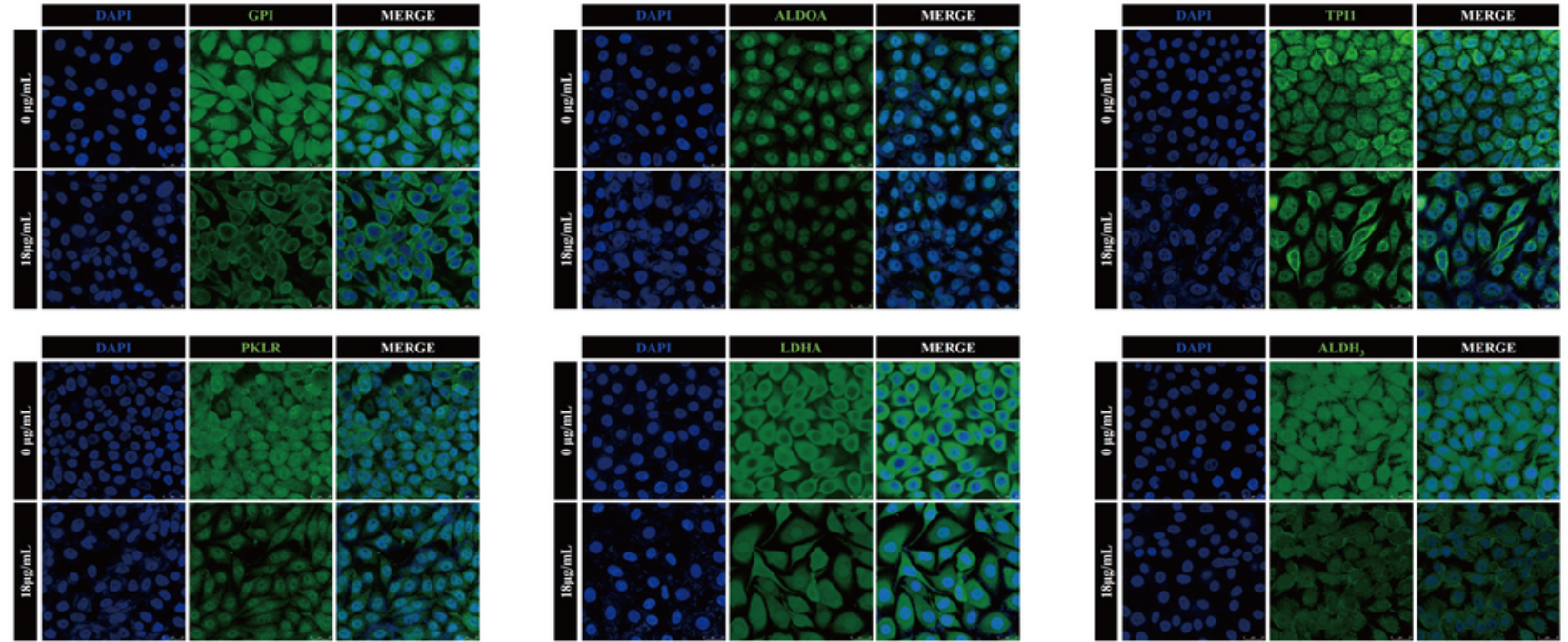

C

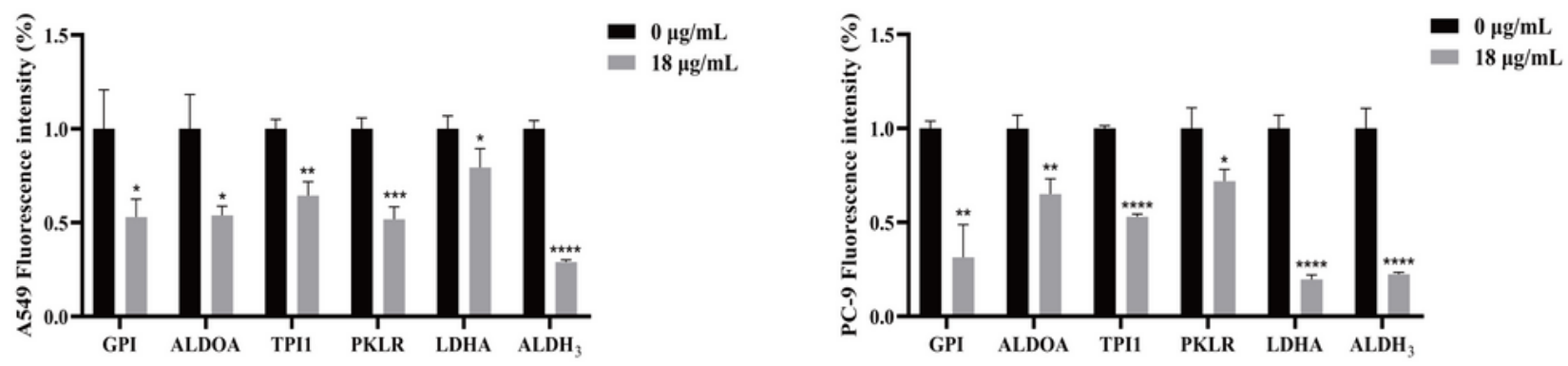

Figure 8

A549and PC-9 cells were treated with a-solanine $(0,12,18,24 \mu \mathrm{g} / \mathrm{mL})$ for $24 \mathrm{~h}$; The proteins expression was detected using a confocal laser confocal microscope. The target proteins presented as green and the nuclei as blue using DAPI counterstaining. Fluorescence intensity was detected using Image $\mathrm{J}$ software. (A) The proteins to be detected for A549. (B) The targets proteins for PC-9. (C) The statistical graphs for 
fluorescence intensity of A549 and PC-9 cells.Full-length blots are presented in Supplementary Figure S3. ${ }^{\star} \mathrm{P}<0.05,{ }^{*} \mathrm{P}<0.01,{ }^{*} * \mathrm{P}<0.001,{ }^{*} * \star \mathrm{P}<0.0001$ versus the $0 \mu \mathrm{g} / \mathrm{mL}$ a-solanine group.

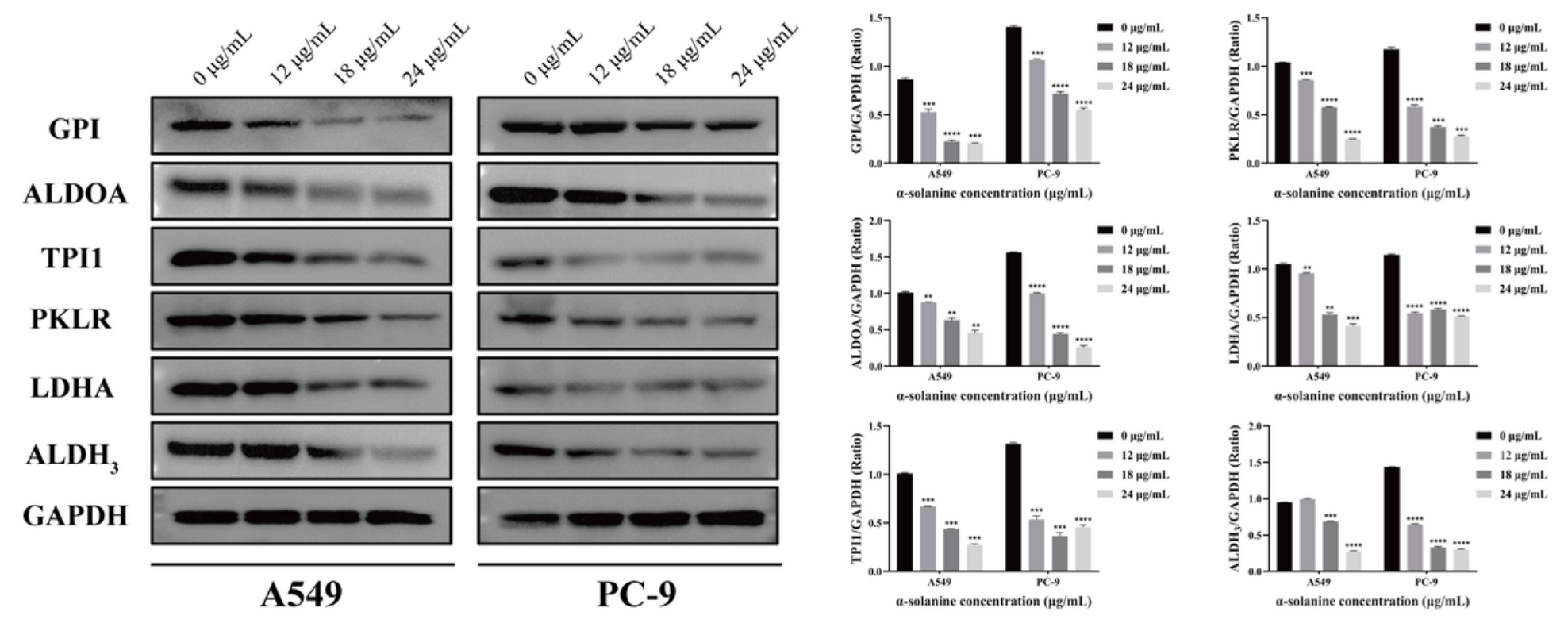

Figure 9

Effect of a-solanineon the expression of GPI, ALDOA, TPI1, PKLR, LDHA and ALDH3 in A549 and PC-9 cells. A549 and PC-9 were treated with various dose of a-solanine for $24 \mathrm{~h}$. And quantification of the densitometric results were performed by calculating the ratio of the control group. ${ }^{*} P<0.05,{ }^{*} P<0.01$, ${ }^{*} * * P<0.001, * \star * * P<0.0001$ versus the $0 \mu \mathrm{g} / \mathrm{mL}$ a-solanine group. 


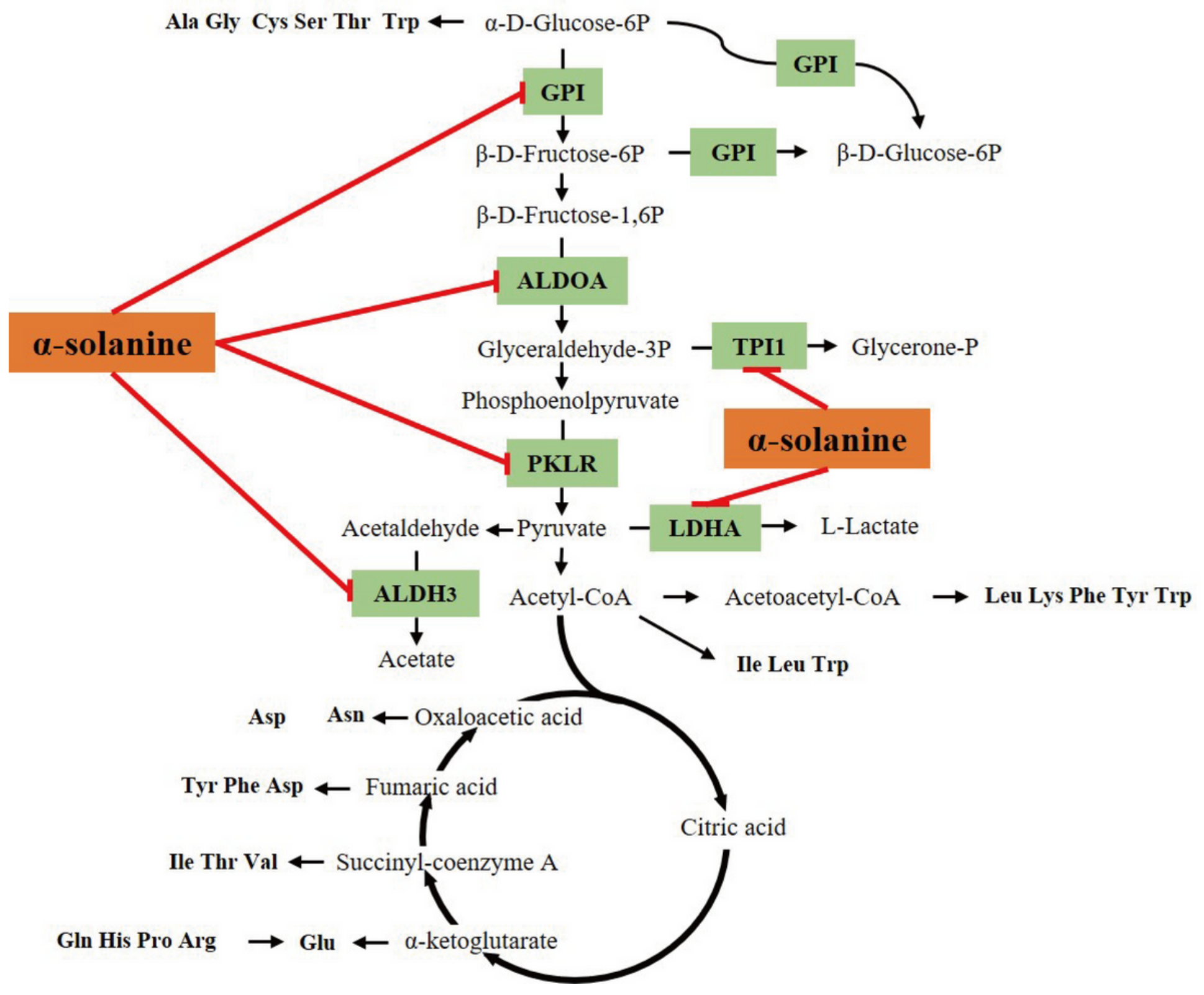

Figure 10

The schematic diagram of the inhibition mechanism of a-solanine on NSCLC.

\section{Supplementary Files}

This is a list of supplementary files associated with this preprint. Click to download.

- SupplementFigureS1.tif

- SupplementFigureS2.tif

- SupplementaryfigureS3.pdf

- SupplementTables1.csv

- SupplementTableS2.csv 
- SupplementTableS3.csv

- SupplementTableS4.csv

- FigureandTableLegents.pdf 